\title{
EFECTO DE LA DENSIDAD DE PLANTACIÓN EN EL CRECIMIENTO DE CUATROS ESPECIES FORESTALES TROPICALES
}

\author{
Pedro Suatunce Cunuhay ${ }^{1,2}$, Gorki Díaz Coronel ${ }^{1,3}$ y ${ }^{\bullet}$ Luz García Cruzatty ${ }^{1}$ \\ ${ }^{1}$ Unidad de Investigación Cientifica y Tecnológica, Universidad Técnica Estatal de Quevedo, $\mathrm{km} 7$ vía \\ Quevedo - El Empalme, C. P. 73. Mocache,Los Ríos, Ecuador. 1 luzcecilia29@hotmail.com \\ ${ }^{2}$ Facultad de Ciencias Ambientales, Universidad Técnica Estatal de Quevedo, km 1 1 1/2 vía a Santo Domingo \\ de los Tsáchilas, C. P. 73. Quevedo, Los Ríos, Ecuador. \\ ${ }^{3}$ Facultad de Ciencias Pecuarias, Universidad Técnica Estatal de Quevedo, km 7 vía a El Empalme \\ Mocache, Los Ríos, Ecuador
}

\begin{abstract}
RESUMEN
Se evaluó el efecto de la densidad en el desarrollo de Sárboles de Tectona grandis L.F., Cordia megalantha S.F. Blake, Cybistax donnell smithii Rose y Triplaris cumingiana Fisch. \& Mey. en parcelas permanentes establecidas en 1997 en la Finca "La Represa". El mayor crecimiento en diámetro se obtuvo a la densidad de 123 árboles ha-1 (espaciamiento: 9 × $9 \mathrm{~m}$ ). La especie que alcanzó mayor crecimiento diamétrico en este espaciamiento fue la Teca con $36.53 \mathrm{~cm}$, seguida por el Guayacán con $33.80 \mathrm{~cm}$. Los árboles registraron un mayor volumen promedio $\left(\mathrm{m}^{3}\right)$ en el espaciamiento $9 \times 9 \mathrm{~m}$. Sin embargo, en las cuatro especies estudiadas hay un mayor volumen por hectárea a un espaciamiento de $6 \mathrm{x}$ $6 \mathrm{~m}$, debido al mayor número de árboles por hectárea. La especie que presentó mayor volumen promedio fue la Teca con $1.076 \mathrm{~m}^{3} \mathrm{y}$ el menor volumen promedio se registró en Laurel con $0.316 \mathrm{~m}^{3}$.
\end{abstract}

Palabras claves: Tectona grandis L.F., densidad, Cordia megalantha S.F. Blake, diámetro, Cybistax donnell smithii Rose y Triplaris cumingiana Fisch. \& Mey.

\begin{abstract}
The effect of the density was evaluated in the deve1 lopment of trees of Tectona grandis L.F., Cordia megalantha S.F. Blake, Cybistax donnell smithii Rose and Triplaris cumingiana Fisch. \& Mey. in permanent parcels established in 1997, in "La Represa" farm. The major growth in diameter was obtained to the density of 123 trees ha ${ }^{-1}$ (spacing: $9 \times 9 \mathrm{~m}$ ). The species that reached major diametric growth in this spacing was the Teak with $36.53 \mathrm{~cm}$, continued by Guayacan with 33.80 $\mathrm{cm}$. The trees registered a major volume average $\left(\mathrm{m}^{3}\right)$ in the spacing $9 \times 9 \mathrm{~m}$. However, in the four studied species there is a major volume for hectare at the spacing of $6 \times 6 \mathrm{~m}$, due to the major number of trees for hectare. The species that presented major volume average was the Teak with $1.076 \mathrm{~m}^{3}$ and the minor volume average registered in Laurel with $0.316 \mathrm{~m}^{3}$.
\end{abstract}

Key words: Tectona grandis L.F., density, Cordia megalantha S.F. Blake, diámetro, Cybistax donnell smithii Rose y Triplaris cumingiana Fisch. \& Mey.

\section{INTRODUCCIÓN}

$\mathrm{E}_{\mathrm{p}}^{\mathrm{n}}$ el litoral ecuatoriano las especies de mayor importancia comercial utilizadas en las plantaciones, son: Cordia alliodora (Ruiz \& Pav.) Oken, Inga sp., Swietenia macrophylla King, Ochroma lagopus Sw., Triplaris cumingiana Fisch. \& C.A. Mey. ex C.A. Mey, Prosopis juliflora (Sw.) DC., Tectona grandis L.F., C. Cybistax donnell smithii Rose (Rizzo, 2005). Los principales motivos para que los reforestadores busquen una mayor producción de madera son los buenos precios en el mercado nacional e internacional y la oportunidad de reducir la diferencia entre la oferta y la demanda.

La silvicultura ha desarrollado una serie de técnicas para lograr un incremento considerable en la producción forestal, tanto en calidad como en cantidad. Dentro de éstas podemos mencionar: el control de malezas, deshijes, fertilización, podas y el control de la densidad; siendo este último uno de los aspectos más relevantes para el desarrollo de los árboles (Arce y Fonseca, 2003).

Recibido: Junio, 2009. Aceptado: Diciembre, 2009.

Publicado como ARTÍCULO en Ciencia y Tecnología 3(1): 23-26. 2010
La manipulación de la densidad es la herramienta más eficiente que dispone el silvicultor para lograr el principal objetivo del manejo de las plantaciones forestales, que es aumentar la cantidad de madera comercial y mejorar la calidad de la misma. El nivel de espesura de un rodal afecta muchas características del mismo; tales como diámetro, altura, volumen, tamaño de copa, vigor de los árboles e incluso la resistencia a plagas y enfermedades (Pérez et al., 2005).

La elección de la densidad de plantación es de máxima importancia. Desde el punto de vista económico, la densidad se decidirá en función del costo y del objetivo de la plantación, con mayores densidades cuando se quiere obtener fustes rectos, y densidades menores cuando se quiere árboles de copas amplias y bien iluminadas para la producción de frutos.

Por los antecedentes expuestos la Universidad Técnica Estatal de Quevedo estableció un ensayo, para determinar el efecto de la densidad de plantación en el crecimiento, de Tectona grandis L.F., Cordia megalantha S.F. Blake, Cybistax donnell smithii Rose y Tripla- 
ris cumingiana Fisch. \& Mey. con base en los resultados de esta investigación los productores forestales podrán realizar manejo de la densidad en sus plantaciones, con un criterio técnico adecuado a sus objetivos de producción.

\section{Materiales y Métodos}

$\mathrm{L}$ a presente investigación se realizó en parcelas experimentales de la Finca Experimental "La Represa", de la Universidad Técnica Estatal de Quevedo. Esta área se encuentra entre las coordenadas geográficas: $01^{\circ}$ 03' 24" de latitud sur y $79^{\circ} 24^{\prime} 55^{\prime \prime}$ de longitud oeste. Pertenece a la formación ecológica bosque húmedo tropical (Holdrige, 1987).

El proyecto se inició en mayo de 1997. La información analizada en esta investigación corresponde a datos obtenidos en el año 2007.

\section{Tratamientos}

Se evaluó dos densidades de plantación en cuatro especies forestales: Tectona grandis L.F., Cordia megalantha S.F. Blake, Cybistax donnell smithii Rose y Triplaris cumingiana Fisch. \& Mey. Los tratamientos evaluados y parcelas están representados en el siguiente esquema:

\section{Diseño experimental}

Las especies forestales se encuentran establecidas bajo un diseño de bloques al azar con arreglo de parcelas divididas y tres repeticiones (Cuadro 1). Los datos fueron analizados mediante el programa estadístico SAS (2002). Para la comparación de medias se utilizó la prueba de rangos múltiples de Duncan $(\mathrm{P} \leq 0.05)$ (Padrón, 1996).

Cuadro 1. Tratamientos y unidades experimentales

\begin{tabular}{cccccc}
\hline \multirow{2}{*}{ Tratamientos } & \multicolumn{5}{c}{ Bloques } \\
\cline { 2 - 6 } & Especies & Distancia (m) & I & II & III \\
\hline 1 & T. grandis & $6 \times 6$ & $\mathrm{~d}_{11}$ & $\mathrm{~d}_{12}$ & $\mathrm{~d}_{13}$ \\
2 & T. grandis & $9 \times 9$ & $\mathrm{~d}_{21}$ & $\mathrm{~d}_{22}$ & $\mathrm{~d}_{23}$ \\
\hline 3 & C. megalantha & $6 \times 6$ & $\mathrm{~d}_{31}$ & $\mathrm{~d}_{32}$ & $\mathrm{~d}_{33}$ \\
4 & C. megalantha & $9 \times 9$ & $\mathrm{~d}_{41}$ & $\mathrm{~d}_{42}$ & $\mathrm{~d}_{43}$ \\
\hline 5 & C. donnell-smithii & $6 \times 6$ & $\mathrm{~d}_{51}$ & $\mathrm{~d}_{52}$ & $\mathrm{~d}_{53}$ \\
6 & C. donnell-smithii & $9 \times 9$ & $\mathrm{~d}_{61}$ & $\mathrm{~d}_{62}$ & $\mathrm{~d}_{63}$ \\
\hline 7 & T. cumingiana & $6 \times 6$ & $\mathrm{~d}_{71}$ & $\mathrm{~d}_{72}$ & $\mathrm{~d}_{73}$ \\
8 & T. cumingiana & $9 \times 9$ & $\mathrm{~d}_{81}$ & $\mathrm{~d}_{82}$ & $\mathrm{~d}_{83}$ \\
\hline
\end{tabular}

\section{Características de las parcelas experimentales}

Las parcelas experimentales establecidas bajo el espaciamiento de $9 \times 9 \mathrm{~m}$, tienen un área total de 972 $\mathrm{m}^{2}$ con 12 árboles. Estos árboles fueron plantados inicialmente en sistema agroforestal con café; habiéndose eliminado esta especie agrícola a los 7 años de edad de la plantación. El número de unidades experimentales evaluadas es de 24 árboles.

Las parcelas estudiadas cuyos árboles se encuentran a una densidad de $6 \times 6 \mathrm{~m}$, tienen un área total de $324 \mathrm{~m}^{2}$ con 9 árboles. Esta plantación fue establecida inicialmente a $3 \times 3 \mathrm{~m}$, habiéndose reducido la densidad a los 7 años de edad mediante un raleo sistemático. El número de unidades experimentales evaluadas fue de 12 árboles.
La población está constituida de 252 árboles. Se evaluaron un total de 36 árboles.

\section{Manejo del experimento}

El manejo silvicultural de los rodales se efectuó de acuerdo a las recomendaciones para cada especie. Se realizaron podas de mantenimiento y fitosanitarias periódicamente, control de malezas manual y químico, según la incidencia. Los datos de diámetro y altura de los árboles fueron tomados una vez al año, utilizando cinta diamétrica e hipsómetro de Sunnto, respectivamente (Diéguez, 2000). 


\section{Resultados y Discusión}

Diámetro y altura de cuatro especies forestales a los diez años

T a altura no presentó diferencias significativas entre Llas densidades, pero sí entre especies. La Teca ( $T$. grandis) obtuvo el mayor crecimiento en altura, en el espaciamiento $6 \times 6$ m; mientras el Guayacán blanco $(C$. donnell-smithii) obtuvo el mayor crecimiento en altura, en la distancia $9 \times 9 \mathrm{~m}$.

El diámetro presentó diferencias significativas entre densidades y entre especies. El mayor diámetro se obtuvo en la distancia 9 × 9 m. La especie que alcanzó mayor crecimiento diamétrico fue la Teca (T. grandis), seguida por Guayacán (C. donnell-smithii) (Cuadro 2).

Cuadro 2. Altura y diámetro a los diez años de edad de cuatro especies forestales establecidas en dos densidades

\begin{tabular}{|c|c|c|c|c|c|c|}
\hline \multirow[b]{2}{*}{ Tratamiento } & \multicolumn{3}{|c|}{ Altura } & \multicolumn{3}{|c|}{ Diámetro } \\
\hline & $\begin{array}{c}\text { Distancia } \\
9 \times 9 \mathrm{~m}\end{array}$ & $\begin{array}{c}\text { Distancia } \\
6 \times 6 \mathrm{~m}\end{array}$ & Promedio & $\begin{array}{c}\text { Distancia } \\
9 \times 9 \mathrm{~m}\end{array}$ & $\begin{array}{c}\text { Distancia } \\
6 \times 6 \mathrm{~m}\end{array}$ & Promedio \\
\hline Guayacán blanco & 20.36 & 18.81 & 19.582 & 33.80 & 24.20 & $29.00 \mathrm{~b}$ \\
\hline Laurel prieto & 15.19 & 15.01 & 15.075 & 22.67 & 17.43 & $20.05 \mathrm{~d}$ \\
\hline Teca & 19.59 & 20.21 & 19.897 & 36.53 & 28.13 & $32.33 \mathrm{a}$ \\
\hline Fernansánchez & 20.20 & 19.44 & 19.817 & 26.10 & 19.60 & $22.85 \mathrm{c}$ \\
\hline Promedio & 18.82 a & $18.36 \mathrm{a}$ & & $29.78 \mathrm{a}$ & $22.34 \mathrm{~b}$ & \\
\hline CV (\%) & & 3.91 & & & 6.07 & \\
\hline
\end{tabular}

Medias con letras iguales no difieren estadisticamente entre sí (Duncan, $\mathrm{P}<0.05$ )

Volumen de cuatro especies forestales a los diez años de edad

A los 10 años de edad, el volumen promedio presentó diferencias significativas entre densidades y entre especies. Los árboles registraron un mayor volu- men promedio $\left(\mathrm{m}^{3}\right)$ en el espaciamiento 9 × $9 \mathrm{~m}$. La especie que presentó mayor volumen promedio fue la Teca (T. grandis) con $1.076 \mathrm{~m}^{3} \mathrm{y}$ el menor volumen promedio se registró en Laurel (C. macrantha) con 0.316 $\mathrm{m}^{3}$ (Cuadro 3).

Cuadro 3. Volumen promedio $\left(\mathrm{m}^{3}\right)$ e incremento medio anual $\left(\mathrm{m}^{3}\right)$ por árbol de cuatro especies forestales, a los diez años de edad, establecidas en dos densidades

\begin{tabular}{|c|c|c|c|c|c|}
\hline \multirow{2}{*}{ Tratamiento } & \multirow{2}{*}{$\begin{array}{l}\text { Distancia } \\
9 \times 9 \mathrm{~m}\end{array}$} & \multirow{2}{*}{$\begin{array}{l}\text { Distancia } \\
6 \times 6 \mathrm{~m}\end{array}$} & \multirow{2}{*}{ Promedio } & \multicolumn{2}{|c|}{ IMA } \\
\hline & & & & $9 \times 9 \mathrm{~m}$ & $6 \times 6 \mathrm{~m}$ \\
\hline Guayacán blanco & 1.188 & 0.571 & 0.879 b & 0.119 & 0.057 \\
\hline Laurel prieto & 0.397 & 0.235 & $0.316 \mathrm{~d}$ & 0.040 & 0.024 \\
\hline Teca & 1.334 & 0.817 & $1.076 \mathrm{a}$ & 0.133 & 0.082 \\
\hline Fernansánchez & 0.707 & 0.382 & 0.545 c & 0.071 & 0.038 \\
\hline Promedio & $0.907 \mathbf{a}$ & $0.501 \mathrm{~b}$ & & & \\
\hline$\overline{C V(\%)}$ & & & 13.45 & & \\
\hline
\end{tabular}

El volumen por hectárea presentó diferencias significativas entre densidades y entre especies. En las cuatro especies estudiadas hay un mayor volumen por hectárea a un espaciamiento de $6 \times 6 \mathrm{~m}$, debido al mayor número de árboles por hectárea. Los mayores volúmenes por hectárea se registraron en Teca (T. grandis) con un Incremento Medio Anual (IMA) de $22.72 \mathrm{~m}^{3}$ y en Guayacán blanco ( $C$. donnell smithii) con un IMA de $15.87 \mathrm{~m}^{3}$, en las parcelas establecidas bajo el espaciamiento de $9 \times 9 \mathrm{~m}$. El menor volumen por hectárea se obtuvo en Laurel (C. macrantha) con un IMA de 6.53 $\mathrm{m}^{3}$ bajo el mismo espaciamiento (Cuadro 4). 
Cuadro 4. Volumen $\left(\mathrm{m}^{3}\right)$ e incremento medio anual $\left(\mathrm{m}^{3}\right)$ por hectárea de cuatro especies forestales, a los diez años de edad, establecidas en dos densidades

\begin{tabular}{|c|c|c|c|c|c|}
\hline \multirow{2}{*}{ Tratamiento } & \multirow{2}{*}{$\begin{array}{l}\text { Distancia } \\
9 \times 9 \mathrm{~m}\end{array}$} & \multirow{2}{*}{$\begin{array}{c}\text { Distancia } \\
6 \times 6 \mathrm{~m}\end{array}$} & \multirow{2}{*}{ Promedio } & \multicolumn{2}{|c|}{ IMA } \\
\hline & & & & $9 \times 9 \mathrm{~m}$ & $6 \times 6 \mathrm{~m}$ \\
\hline Guayacán blanco & 146.124 & 158.675 & $152.40 \mathrm{~b}$ & 14.61 & 15.87 \\
\hline Laurel prieto & 48.837 & 65.282 & $57.06 \mathrm{~d}$ & 4.88 & 6.53 \\
\hline Teca & 164.118 & 227.215 & $195.67 \mathrm{a}$ & 16.41 & 22.72 \\
\hline Fernansánchez & 86.978 & 106.115 & $96.58 \mathrm{c}$ & 8.70 & 10.61 \\
\hline Promedio & 111.514 & 139.34 & & & \\
\hline CV (\%) & & & 16.22 & & \\
\hline
\end{tabular}

Medias con letras iguales no difieren estadisticamente entre sí (Duncan, $\mathrm{P}<0.05$ )

El Ministerio de Ambiente ha reportado en Teca (T. grandis) un volumen por hectárea de 126.84 $\mathrm{m}^{3}$, a una densidad de 200 árboles por hectárea $(10 \mathrm{x}$ $5 \mathrm{~m}$ ) y en Guayacán blanco (C. donnell smithii) un volumen de $268 \mathrm{~m}^{3}$, a una densidad de 400 árboles por hectárea $(5 \times 5 \mathrm{~m})$, a los 11 años de edad. Este volumen registrado en Teca (T. grandis) es inferior al alcanzado bajo las dos densidades evaluadas en el presente estudio. Mientras que el volumen indicado en Guayacán supera a los volúmenes obtenidos bajo las densidades de siembra estudiadas (Fernández, 2000).

\section{Conclusiones}

A los diez años de edad, las especies establecidas a 9 x $9 \mathrm{~m}$ tuvieron un mayor crecimiento en diámetro, y el crecimiento en altura fue similar en las dos densidades.

La especie que presentó mayor crecimiento en diámetro y altura fue la Teca (T. grandis). La especie que obtuvo menor crecimiento en diámetro fue el Laurel (C. macrantha) y la que registró menor crecimiento en altura fue el Fernansánchez (T. cumingiana).

Las especies forestales obtuvieron los mejores promedios de volumen por árbol en la distancia 9 x 9 $\mathrm{m}$, sin embargo el volumen de madera por hectárea es mayor a $6 \times 6 \mathrm{~m}$, por la mayor cantidad de árboles por hectárea.

La teca ( $T$. grandis) y el Guayacán ( $C$. donnell smithii) obtuvieron mayores volúmenes de madera bajo los dos espaciamientos evaluados.

El mayor IMA por individuo se obtuvo a una densidad de 123 árboles por hectárea $(9 \times 9 \mathrm{~m})$, pero el mayor IMA por hectárea se consiguió a una densidad de 277 árboles (6 x $6 \mathrm{~m})$.

\section{Literatura Citada}

Arce,V., W. Fonseca. 2003. Relación albura-duramen y características físicas de la madera Tectona grandis en plantaciones de 10 años con diferente densidad de siembra. Guanacoste - Costa Rica. Consultado el 22 de julio del 2007. Disponible en: www.varce. pdf

Diéguez, V. 2000. Dendrometría. España. Editorial Mundi Prensa.

Fernández, M. 2000. Factores de Emisión en el sector cambio del uso del suelo y silvicultura. Ministerio de Ambiente del Ecuador. Cambio Climático. 50 p.

Holdrige, L. 1987. Ecología basada en zonas de vida IICA. San José, Costa Rica. 216 p.

Padrón, E. 1996. Diseños Experimentales con aplicación a la agricultura y la ganadería. México. Trillas. $215 \mathrm{p}$.

Pérez, M., J. Carew y N. Battey. 2005. Efecto de la densidad de plantación sobre el crecimiento vegetativo y reproductivo de la Fresa cv. Elsanta. Bioagro. Vol. 17 (1): 11-15.

Rizzo, P. 2005. Especies maderables. Servicio de Información Agropecuaria del Ministerio de agricultura y Ganadería del Ecuador. Consultado el 03 de agosto del 2007. Disponible en www.especies maderables.htm.

SAS. 2002. SAS. User's guide: Statistics. Versión 9.0 Statistical Analysis Sistem Institute. Cary, NC. 